\title{
DYNAMICAL MODELS OF ELLIPTICAL GALAXIES
}

\author{
C. P. WILSON \\ Hale Observatories, Carnegie Institution of Washington, California Institute of Technology, \\ Pasadena, Calif., U.S.A.
}

\begin{abstract}
Self-consistent dynamical models of elliptical galaxies have been constructed using a modification of the Prendergast-Tomer algorithm. They reproduce many of the observed properties of elliptical galaxies.
\end{abstract}

Because the relaxation time of an elliptical galaxy is greater than its age, such a system is at present in a stationary configuration. Consequently, we have attempted to represent elliptical galaxies by self-consistent models which are time-independent, rotationally symmetrical, and rotating.

The algorithm for constructing a model is very similar to that used by Prendergast and Tomer (1970): one adopts a distribution function $f(E, J)$ that depends on the integrals of motion (the energy and the component of angular momentum parallel to the axis of symmetry), integrates this function over velocities to get the density, and then solves Poisson's equation. Since the theory of the initial collapse process (e.g. Lynden-Bell, 1967) is not very rigorous, the choice of a distribution function is somewhat arbitrary. The following function has been used here because it includes the necessary degrees of freedom in a simple and reasonable fashion:

$$
\begin{array}{rlrl}
f(E, J) & =\left(e^{-E}-1+E\right) \exp \left(\beta J-\frac{1}{2} \zeta^{2} J^{2}\right), & & E<0 . \\
& =0 \quad, \quad E \geqslant 0 .
\end{array}
$$

The free parameters $\beta$ and $\zeta$ permit construction of a range of models. Except for a modification of the energy dependence, which makes $f$ go to zero continuously and smoothly at $E=0$, this is simply a classical distribution function that is quadratic in the velocities (Eddington, 1915, and others). To solve Poisson's equation, the density and potential are expanded into Legendre series with coefficients which are functions of radius. The resulting simultaneous set of integral equations are solved numerically (Wilson, 1973).

Models have been projected onto the plane of the sky and compared with observations of NGC 3379. If the inclination of this galaxy is assumed to lie anywhere between $45^{\circ}$ (with intrinsic axial ratio 0.6 ) and $90^{\circ}$ (intrinsic axial ratio 0.85 ), a model can be found that represents rather well the observed variations of surface brightness and isophote shape with radius (Miller and Prendergast, 1962). These models also agree with the observed velocity dispersion (Burbidge et al., 1961) and rotation curve (Peterson, 1974) within the large observational uncertainties.

All elliptical galaxies have similar radial intensity profiles which the models fit fairly well. However, the great observed variety of the flattening vs. radius curves cannot be reproduced; the models all start nearly round at the center, increase to a 
maximum flattening at some intermediate radius, and then become nearly round again at the boundary.

Once a dynamical model has been fitted to the photometric and kinematic properties of a galaxy, it can predict such interesting parameters as the mass-to-light ratio. For NGC 3379, we have $(\mathfrak{M} / L)_{v}=3.3$ (solar units, $H=55 \mathrm{~km} \mathrm{~s}^{-1} \mathrm{Mpc}^{-1}$ ). The limited accuracy of observations introduces an uncertainty of a factor of two into this value, whereas the unknown inclination and fitting to different models results in only a $5 \%$ uncertainty. The same data analyzed in the conventional manner (Morton and Chevalier, 1972), including several questionable assumptions, gives $(\mathfrak{M} / L)_{v}=9.6$. The importance of a proper dynamical treatment of the data is obvious.

By using the stability criterion proposed by Ostriker and Peebles (1973), it is found that only the models with peak flattening less than E4 to E5 are stable.

\section{References}

Burbidge, E. M., Burbidge, G. R., and Fish, R. A.: 1961, Astrophys. J. 134, 251.

Eddington, A. S.: 1915, Monthly Notices Roy. Astron. Soc. 76, 37.

Lynden-Bell, D.: 1967, Monthly Notices Roy. Astron. Soc. 136, 101.

Miller, R. H. and Prendergast, K. H.: 1962, Astrophys. J. 136, 713.

Morton, D. C. and Chevalier, R. A. : 1972, Astrophys. J. 174, 489.

Ostriker, J. P. and Peebles, P. J. E. : 1973, Astrophys. J. 186, 467.

Peterson, C. J.: 1974, private communication.

Prendergast, K. H. and Tomer, E.: 1970, Astron. J. 75, 674.

Wilson, C. P.: 1973, 'Dynamical Models of Elliptical Galaxies', University of California, Berkeley (Ph.D.

Thesis).

\section{DISCUSSION}

Freeman: Can you think of any way to tell whether ellipticals are axisymmetric or genuinely triaxial?

Wilson: I don't know of any definite observational test. It is instructive to consider obviously nonaxisymmetric systems, e.g. SBO's. One property in which they differ from ellipticals is that the position angle of the major axis of the isophotes varies with radius. Perhaps the constant position angle in ellipticals is telling us something about this problem. 